\title{
Pensieri in una notte di picchetto nel Malcantone
}

\author{
M. Bernasconi-Sciaroni
}

È una domenica notte. È novembre. Sono passate da poco le due. Una chiamata: al telefono la voce un po' concitata di una donna. Suo padre malato di Alzheimer si è svegliato agitato e confuso: cammina per la casa gridando. Hanno già provato a calmarlo. Non ce la fanno. Sì, è già successo altre volte. Vorrebbero un medico in casa. Dietro la voce della donna una gran confusione. Che consigli potrei mai dare al telefono? Parto. Non ho scelta.

Pioviggina. Alla rotonda di Caslano devo imboccare la strada per Pura e poi continuare a salire fino al bivio prima di Curio. Qui dovrò svoltare a sinistra e continuare in direzione di Bedigliora. Accendo la radio per rilassarmi. Non riesco ad ascoltarla. Anche stavolta mi prende una specie di magone e non so bene perché. È forse il retaggio delle prime guardie di assistente? Eravamo soli per tutto l'ospedale. In pronto soccorso una o due infermiere che allora mi sembravano ostili.

Solitudine.

La strada sale a curvoni. È molto buio e ci sono lunghi tratti nei boschi. Una patina di foglie bagnate sull'asfalto. E se rimanessi qui in panne? A metà di un tornante un cane immobile, abbagliato dai fari, mi fissa; stava rovistando nell'immondizia.

La notte precedente, quella di sabato, sono stata fortunata. Nemmeno una telefonata. Di giorno qualche consiglio al telefono e poche visite, mi pare per febbre. Non me le ricordo già quasi più. Mi ritorna in mente invece l'ultimo paziente visto venerdì in studio poco prima che chiudessi. Un giovanotto di solito chiacchierone e baldanzoso. Venerdì parlava poco e aveva un colorito strano. Era venuto per dolori alla pancia. Per nessuna ragione era disposto a passare così il fine settimana. Voleva una ricetta!

Gli addomi sono dei misteri.

Aveva accettato con riluttanza di essere esaminato. Lasciavo che le mani sentissero e premessero quel ventre un po' gonfio, e intanto mi dicevo: «Mani! Parlatemi anche stavolta! Ditemi cosa devo fare.» E ora mentre guido mi chiedo perché Claude Luezior, che ha parlato di tante mani, non ha parlato di quelle di un povero medico di famiglia. Non sono forse mani che devono imparare a parlare per dire: «Mandalo in ospedale!». O per dire: «Lascialo a casa.» Venerdì le avevo ascoltate, avevo obbedito e inviato il ragazzo in ospedale.

Avevo poi saputo che poco dopo iniziava per lui il gran cerimoniale della sala operatoria.

E se non l'avessi mandato? Chissà se anche stavolta avrei udito e ascoltato quella vocina imperiosa che da non so quale angolo remoto mi avrebbe ordinato: «Nel dubbio mandalo!».

Sono al bivio. Ho imboccato la strada giusta. Non c'è in giro anima viva. Nemmeno un'automobile. Nessuna finestra accesa nelle poche case lungo la strada. È ora che cominci a ordinare le idee. Che cosa mi aspetta in quella casa? Meglio prepararmi al peggio. Potrebbe esserci un gran caos. Spero che ci saranno poche persone. Confesso che mi dà un certo fastidio sentirmi osservata mentre sono intenta nel mio lavoro. Figuriamoci poi se su qualche comodino traballante ingombro di scatoline di medicamenti, di bicchieri, di foto incorniciate e alla luce fioca di qualche lampadina sono costretta ad esempio a preparare delle iniezioni. L'ultima volta non riuscivo a spezzare una fialetta: si sentiva soltanto lo stridio della mia seghetta e il fiato sospeso dei parenti attorno al letto.

Ma cosa mai potrebbe avere questo povero vecchio in una notte cosi? Quali mai potrebbero essere le cause di un delirio di questo genere? Non si era forse posto una domanda quasi uguale il dottor Bovary, tanti anni fa? L'avevano chiamato in piena notte per soccorrere un agricoltore che si era fratturato una gamba. Mentre viaggiava sul calesse ripassava mentalmente quello che sapeva sulle fratture e fu sollevato quando vide che si trattava di una frattura semplice! Grazie Flaubert! Eri figlio di un medico e hai saputo ascoltare e capire i suoi dubbi e le sue debolezze.

Se avrò un po' di fortuna anch'io, il mio paziente si sarà già calmato almeno un po' prima che arrivi. Però questi casi psichici sono forse i più terribili. L'anno scorso una giovane donna in pieno scompenso mi aveva scaraventato addosso una bibbia e stava per fare la stessa cosa del suo bebè. Sono tremendi i ricoveri coatti. L'ambulanza. La polizia. Violenza e disperazione. Non voglio che anche stanotte finisca così. In fondo è passato poco tempo da quando mi sono trovata 
in mezzo al lampeggiare sinistro delle auto della polizia. Era una sera di settembre o ottobre, mi ricordo che fuori era ancora tiepido. Un uomo si era buttato sotto le ruote del treno a Magliaso. Un miserabile fagotto di carne appiccicata alla ruota. Attorno il cordone bianco e rosso e silenzio. Pochi curiosi. Nessuno lo piangeva, uno straniero arrivato da poco.

Quanto diverso, quanto più consolante era stato invece, pochi giorni dopo, certificare la morte di una donna ammalata che aspettava da tempo nel suo letto di poter morire.

Ormai mancheranno pochi chilometri alla meta. Certo, sarei più sollevata se fossi con qualcuno. Perché non fare i picchetti con un collega o con uno studente che di notte potrebbe chiudere i suoi libroni e guardare la realtà? Era stata saggia la fata Turchina a chiamare al capezzale di Pinocchio tre dottori assieme! C'erano nientemeno che il Gufo, la Civetta e il Grillo parlante. Continua a piovere e c'è poca visibilità.

Quanto rimpiango, mentre sto guidando, la mia routine in studio! Il chiacchiericcio dei pazienti in sala d'aspetto, la voce gentile della segretaria che risponde al telefono, il plichetto di ricette pronte da firmare, le cartelle del giorno ammucchiate con ordine sulla scrivania e magari il rumore secco delle Ketalgin che ad una ad una l'aiuto-medico lascia cadere nel bicchiere di cartone per i miei «tossico». Me li immagino appoggiati al banco a scambiare con lei due parole. Intanto passerei in rivista i nomi dei pazienti sulle cartelle. Riconoscerei i fedeli: i fedeli simpatici e i fedeli un po' noiosi. I nuovi arrivi. Qualche traditore, forse reo confesso: non sempre. Riconoscerei anche i nomi dei pazienti con i quali nel corso degli anni è nata quasi un'amicizia segreta e inconfessata. Amici che si vedono solo lì, in studio, e dei quali non si parla a nessuno. Conosco i loro segreti e li custodisco.

Ora vedo che c'è un uomo sulla strada con l'ombrello aperto. Sta gesticolando. Sono arrivata. Mi fa strada. Ha una torcia perché passiamo attraverso i cortili interni di una vecchia casa. Saliamo per una scaletta e siamo in un vecchio tinello. C'è molta gente, ma sono tutti calmi. Il povero vecchio si è quietato di colpo prima che arrivassi. È piuttosto alto e ossuto e mi guarda un po' stupito, con uno sguardo mite. La moglie mi sembra sfinita. Si è infilata un grembiule sopra la camicia da notte. Attorno sei o sette persone tra figli, generi e nuore. Con le loro scarpe da tennis, il maglione e i jeans mi sembrano una squadra di sportivi.

Gli parlo. Lo esamino. Indossa un pigiama a righe. Ma cosa potrei mai scoprire? Come potrei sondare i misteri di un cervello con il mio umile amico stetoscopio che giace arrotolato e inutile sulle mie ginocchia e con le due o tre lampadine che ho nella borsa?

Sì, certo: un tè potrebbe fargli bene. Menta o verbena? È uguale. Qualcuno corre in cucina a prepararlo. Sì, mi dicono, il loro medico sa tutto e ha già proposto che lo si mandi al ricovero per dar sollievo alla moglie, ma lei non si decide.

È cosi stanca, poveretta. Non potrà avere una vecchiaia tranquilla, lei che ha a che fare con la follia di suo marito. Uguale a prima a guardarlo, ma ugualmente cosi diverso ed estraneo. E a volte pauroso. Lui, sempre stato così cortese.

Mi congedo e parto. Un membro della squadra mi scorta fino all'auto, insiste per portarmi la borsa. Si sbraccia mentre manovro in retromarcia. Ringrazia ancora e in fondo non ho fatto niente. Imbocco la strada in discesa. Mi sento più leggera. L'auto corre un po' più veloce, il cane non c'è più, ma ha lasciato un gran disordine di sacchetti stracciati. Daranno la colpa a qualche gatto randagio.

Ora vedo dall'alto le lucine rosse e verdi dell'aeroporto di Agno.

Ripensando al mio vecchio e alla sua tribù, mi dico che forse il nostro lavoro, in questo mondo moderno, non è del tutto inutile.

E mi dico: metti che un giorno toccherà a un tuo figlio star male di notte, in qualche lontana cameretta. Magari in Australia! Troverà sul suo calepino un numero da comporre. Non sarà il 144. Ma ci sarà. E anche lì in qualche altra stanza un medico stanco si trascinerà fuori dal letto. Si metterà al volante e cercherà il suo indirizzo. Suonerà alla sua porta con la borsa in una mano e il cellulare nella tasca della giacca. Gli chiederà bruscamente perché mai l'ha disturbato a quell'ora. Ma poi siederà su una sedia accanto al suo letto e sarà lì in quel momento solo per lui. Magari gli lascerà anche il suo recapito e se il giorno dopo non starà meglio, vedranno poi assieme come andare avanti.

Sono le tre e mezza passate. Tra non molto si incammineranno verso l'aeroporto le donne che iniziano il primo turno. Ne conosco alcune che preparano i panini per i voli della giornata perché sono mie pazienti: due hanno una tendinite al polso. Chissà come se la caveranno oggi a farcire quelle centinaia di panini prima delle sei. A quell'ora tutto dev'essere pronto per il decollo dei primi voli per Zurigo e per Ginevra.

Ora non mi rimane che imboccare la strada in direzione di Lugano. Accendo la radio. Stanno trasmettendo una canzonetta anni sessanta. Non potevo augurarmi di meglio. 